\title{
Subtraction of power counting breaking terms in chiral perturbation theory: spinless matter fields
}

\author{
Meng-Lin Du, ${ }^{a}$ Feng-Kun Guo ${ }^{b}$ and Ulf-G. Meißner ${ }^{a, c}$ \\ ${ }^{a}$ Helmholtz-Institut für Strahlen- und Kernphysik and Bethe Center for Theoretical Physics, \\ Universität Bonn, \\ Nußallee 14-16, D-53115 Bonn, Germany \\ ${ }^{b}$ CAS Key Laboratory of Theoretical Physics, Institute of Theoretical Physics, \\ Chinese Academy of Sciences, \\ Zhong Guan Cun East Street 55, Beijing 100190, China \\ ${ }^{c}$ Institute for Advanced Simulation, Institut für Kernphysik and Jülich Center for Hadron Physics, \\ Forschungszentrum Jülich, \\ Wilhelm-Johnen-Straße, D-52425 Jülich, Germany \\ E-mail: du@hiskp.uni-bonn.de, fkguo@itp.ac.cn, \\ meissner@hiskp.uni-bonn.de
}

ABSTRACT: When matter fields are included in chiral perturbation theory, the nonvanishing mass in the chiral limit introduces a new energy scale so that the loop diagrams including such matter field propagators spoil the usual power counting. However, the power counting breaking terms can be absorbed into counterterms in the chiral Lagrangian. In this paper, we systematically derive these terms to leading one-loop order (next-to-next-to leading order in the chiral expansion) at once by calculating the generating functional using the path integral. They are then absorbed by counterterms in the next-to-leading order Lagrangian. The method can be extended to calculating power counting breaking terms for other matter fields.

KeYwords: Chiral Lagrangians, Effective field theories

ARXIV EPRINT: 1609.06134 


\section{Contents}

1 Introduction 1

2 Power counting and effective Lagrangian 3

$\begin{array}{lll}3 & \text { One-loop generating functional } & 6\end{array}$

4 Subtraction of the power counting breaking terms $\quad 8$

$\begin{array}{lll}5 & \text { Summary } & 10\end{array}$

$\begin{array}{ll}\text { A Kernels } & 11\end{array}$

B Infrared regular parts of loop integrals $\quad 12$

\section{Introduction}

Quantum chromodynamics (QCD) is the fundamental theory for the strong interactions of quarks and gluons. An important feature of QCD is asymptotic freedom, which implies that in the high energy region the observables can be expanded in powers of the strong coupling constant $\alpha_{s}$. However, at low energies, the theory is highly non-perturbative since $\alpha_{s}$ becomes large. The failure of a perturbative expansion in $\alpha_{s}$ requires alternative approaches to investigate the low-energy behavior of the strong interactions. Chiral perturbation theory (ChPT) [1, 3] as the low-energy effective theory of QCD (and the Standard Model) presents a very important tool in this field. It is based on the spontaneous and explicit breaking of chiral symmetry. The massless QCD Lagrangian has a global symmetry $\mathrm{U}(N)_{L} \times \mathrm{U}(N)_{R}$ at the classical level, with $N$ the number of the light quark flavors under consideration. However, the singlet axial current develops an $\mathrm{U}(1)_{A}$ anomaly at the quantum level $[4,5]$. In other words, the QCD Lagrangian has a chiral symmetry $\mathrm{SU}(N)_{L} \times \mathrm{SU}(N)_{R} \times \mathrm{U}(1)_{V}$ in the limit of vanishing quark masses. While the $\mathrm{U}(1)_{V}$ symmetry is manifest as baryon number conservation, the chiral symmetry $\mathrm{SU}(N)_{L} \times \mathrm{SU}(N)_{R}$ is spontaneously broken into its vectorial subgroup $\mathrm{SU}(N)_{V}$, and $N^{2}-1$ Goldstone bosons emerge. Meanwhile, the nonvanishing quark masses break the chiral symmetry explicitly, giving a finite but small mass to the Goldstone bosons. The spontaneously and explicitly broken chiral symmetry plays a central role in the low-energy domain of the strong interactions, and lays the basis for ChPT which is a model-independent effective field theory equivalent to QCD at low energies.

As an effective theory, ChPT provides a powerful and successful framework for studying hadron physics at low energies. It explores a scale separation. The hard energy scale is given by the scale of chiral symmetry breaking $\Lambda_{\chi} \sim 1 \mathrm{GeV}$, which corresponds to the occurrence 
of "typical" hadrons. The soft energy scale is provided by the low-momenta of Goldstone bosons and external fields as well as the Goldstone boson masses (or equivalently the light quark masses). The Goldstone bosons as the low-energy degrees of freedom are treated explicitly, while the high-energy modes are integrated out and the information is encoded into the coupling constants, the so-called low-energy constants (LECs). Due to the small values of the light quark ( $u, d$ and $s$ ) masses, the explicit chiral symmetry breaking effects can be treated perturbatively, and one can construct the chiral Lagrangian order by order in a double expansion in the small momenta, denoted as $p$, of the Goldstone bosons and the light quark masses $m_{q}=\mathcal{O}\left(p^{2}\right)$, where the power of $p$ is called the chiral dimension. Since the effective Lagrangian contains an infinite number of local operators, a proper power counting rule is necessary to determine the relative importance of the various terms and the Feynman diagrams based on them. The power counting [1] for a given term and Feynman diagram may be obtained by rescaling the external momenta $p \rightarrow t p$ and the light quark masses $m_{q} \rightarrow t^{2} m_{q}$ and counting the power of $t$, which gives the chiral dimension. Applying this power counting scheme, a relation between the momentum expansion and the loop expansion is well established for the purely Goldstone boson sector, i.e. when there are no matter fields.

The systematic studies of the purely Goldstone boson ChPT up to one loop, i.e. $\mathcal{O}\left(p^{4}\right)$, have been performed by Gasser and Leutwyler in their classical papers [2,3]. The full generating functional (including one-loop graphs) at $\mathcal{O}\left(\phi^{4}\right)$, i.e. with at most four external Goldstone bosons, was obtained up to $\mathcal{O}\left(p^{4}\right)$, which suffices to calculate all relevant two-point correlation functions. The one-loop diagrams with all vertices from the $\mathcal{O}\left(p^{2}\right)$ Lagrangian appear at the order $\mathcal{O}\left(p^{4}\right)$. They are ultraviolet (UV) divergent and the UV divergences can be renormalized by the counterterms in the $\mathcal{O}\left(p^{4}\right)$ Lagrangian using dimensional regularization which preserves all the symmetries under consideration.

Matter fields which have nonvanishing masses in the chiral limit can be included in ChPT as well. ChPT including light baryons is known as baryon ChPT. There exists a notable power counting breaking (PCB) issue in baryon ChPT [6]: the naive power counting rule does not work for loop diagrams containing baryon propagators and all such diagrams start contributing at $\mathcal{O}\left(p^{2}\right)$ using the $\overline{\mathrm{MS}}$ subtraction scheme of dimensional regularization. ${ }^{1}$ This problem is caused by the nonvanishing mass of matter field in the chiral limit, which then presents an energy scale of $\mathcal{O}(1)$. Various approaches were proposed to address the PCB issue, e.g. the heavy baryon ChPT (HBChPT) $[8,9]$, the infrared regularization (IR) [10], and the extended-on-mass-shell (EOMS) scheme [11]. Likewise, ChPT including other matter fields has the same PCB problem. Note further that the approaches used for baryons can be employed to ChPT including other matter fields.

In $\mathrm{HBChPT}$, the heavy components of the baryon fields are integrated out. The dependence of the matter field mass $m$ is then removed from propagators by expanding the Lagrangian in powers of $1 / \mathrm{m}$. The resulting loops satisfy the power counting. However, the $1 / m$ expansion sometimes produces incorrect low-energy analytic properties [12]. The

\footnotetext{
${ }^{1}$ The closed matter field loops are not taken into account because they are real below the two matter-field threshold and are counted as $\mathcal{O}(1)$. Their contributions can be absorbed by a redefinition of LECs [6, 7].
} 
expansion series near the anomalous threshold fails to converge and an infinite number of terms are needed. ${ }^{2}$ A manifestly Lorentz covariant regularization scheme which preserves the analytic structure and the power counting can also be formulated. A very important step was made by Ellis and Tang $[14,15]$. They noted that the soft-momentum part of a loop diagram is infrared singular and the PCB terms, coming from the hard-momentum modes only, are a local polynomial in small momenta and Goldstone boson masses and can be absorbed into the LECs of the most general chiral Lagrangian. Based on their work, Becher and Leutwyler proposed the IR scheme which isolates the infrared singular parts of the loops by extending the Feynman parameter integration upper bound from unity to infinity [10]. Due to the fact that the infrared regular parts of loops can be obtained by expanding the integrand in small quantities and then integrating each term [16], the EOMS scheme was proposed, in which additional subtractions beyond the $\overline{\mathrm{MS}}$ scheme are performed to get rid of the PCB terms.

In this paper, we will study the one-loop generating functional of correlation functions with up to four external particles for the chiral Lagrangian with spinless matter fields in the fundamental representation of $\mathrm{SU}(N)$. Such a theory can be applied to study kaonpion scattering by treating kaons as matter fields [7], and it is expected to have a better convergence of the chiral expansion than that of the normal SU(3) ChPT which treats kaons as Goldstone bosons as well. It can also be employed to investigate the interaction between heavy mesons and Goldstone bosons $[17,18]$. A systematic one-loop renormalization of this theory was done in ref. [19] by calculating the divergence of the effective action using the background field method and heat kernel technique. This paper presents a step further as we will provide a systematic subtraction of the PCB terms at the Lagrangian level at the leading one-loop order, i.e. $\mathcal{O}\left(p^{3}\right)$, for such a theory. All PCB terms will be obtained at once by calculating the corresponding part in the one-loop generating functional.

This paper is organized as follows. In section 2, the power counting formula and its breaking in the presence of matter fields are briefly introduced; the relevant notation and the chiral Lagrangian for spinless matter fields in the $\mathrm{SU}(N)$ fundamental representation are given to the next-to-next-to-leading order (NNLO). The explicit one-loop generating functional is derived in section 3. In section 4, the PCB terms are obtained and subtracted in the EOMS scheme by redefining the $\mathcal{O}\left(p^{2}\right)$ LECs. Section 5 presents a brief summary.

\section{Power counting and effective Lagrangian}

We denote the matter fields and Goldstone boson fields as $P$ and $\phi$, respectively. To introduce the effective Lagrangian, one has to specify the power counting rules. At low energies, the momenta, as well as the masses $M_{\phi}$, of Goldstone bosons are counted as $\mathcal{O}(p)$. However, the nonvanishing mass of the matter field, $m_{P}$, in the chiral limit introduces a new energy scale. Since matter fields are normal hadrons which are not Goldstone bosons, their masses are of the order of $\Lambda_{\chi}$ and should be counted as $\mathcal{O}(1)$. The temporal component of the momentum of matter field should then be counted in the same way. Yet, at low

\footnotetext{
${ }^{2}$ Note, however, that this can be overcome by using the extended propagator $i /\left(v \cdot k+k^{2} / 2 m\right)$ instead of the strict HB propagator $i / v \cdot k$, see e.g. ref. [13].
} 
energies when the three-momentum of matter field is small, one may count $q^{2}-m_{P}^{2}$ as $\mathcal{O}(p)$, and thus the propagator as $\mathcal{O}\left(p^{-1}\right)$. The Goldstone boson propagator $i /\left(q^{2}-M_{\phi}^{2}\right)$ is counted as $\mathcal{O}\left(p^{-2}\right)$. One can then derive the chiral dimension $n$ for a given Feynman diagram as

$$
n=4 L+\sum_{k} V_{k}-2 I_{\phi}-I_{P}
$$

where $L, V_{k}, I_{\phi}$ and $I_{P}$ denote the numbers of loops, the $k^{\text {th }}$ order vertices, internal Goldstone boson propagators and internal matter field propagators, respectively.

For a specific Feynman graph, if there exist terms whose chiral order is lower than that given by eq. (2.1), those terms are called PCB terms. The power counting given in eq. (2.1) works well and no PCB term exists for purely Goldstone boson ChPT. However, PCB terms show up when matter field propagators enter the loop integrals which are calculated using dimensional regularization with the $\overline{\mathrm{MS}}$ scheme. This is due to the existence of the new energy scale $m_{P}$. It is worth noting that the matter field mass in the chiral limit, denoted as $m$, is of the same chiral order as the physical masses. In the EOMS scheme, these PCB terms are absorbed into the redefinition of the LECs so as to make the amplitudes have a power counting consistent with eq. (2.1).

We only consider the case involving a single matter field. The relevant generating functional is defined as

$$
\left.e^{i Z\left[j, J, J^{\dagger}\right]}=\langle 0 \text { out }| 0 \text { in }\right\rangle_{j, J, J^{\dagger}}=\int[d \phi]\left[d P d P^{\dagger}\right] \exp \left\{i \int d^{4} x\left[\mathcal{L}_{\phi}(j)+\mathcal{L}_{\phi P}\left(J, J^{\dagger}\right)\right]\right\},
$$

where $\mathcal{L}_{\phi}$ and $\mathcal{L}_{\phi P}$ denote the purely Goldstone boson effective Lagrangian and the Lagrangian for the interaction between Goldstone bosons and a single matter field, respectively. Further, $J$ and $J^{\dagger}$ denote the external sources coupled to the matter fields, and $j$ collects various external fields coupled to the Goldstone bosons, i.e. vector $v_{\mu}$, axial-vector $a_{\mu}$, scalar $s$ and pseudoscalar $p$. As usual, the explicit $\mathrm{SU}(N)$ symmetry breaking effect by quark masses $\mathcal{M}$ will be included in the Lagrangian through the external scalar source $s$ via $\chi=2 B_{0} s$, with $s=\mathcal{M}=\operatorname{diag}\left(m_{u}, m_{d}, \ldots\right)$, where $B_{0}$ is a constant related to the quark condensate. The effective Lagrangians can be expanded in a power series as

$$
\mathcal{L}_{\phi}=\sum_{n=1}^{\infty} \mathcal{L}_{\phi}^{(2 n)}, \quad \mathcal{L}_{\phi P}=\sum_{n=1}^{\infty} \mathcal{L}_{\phi P}^{(n)}
$$

where the upper indices indicate the chiral dimensions.

To construct the effective Lagrangians respecting the chiral symmetry, we collect the Goldstone bosons in a $N \times N$ unitary matrix $\mathrm{U}(x)$,

$$
\mathrm{U}(x)=u^{2}(x)=\exp \left(\frac{i \phi}{F_{0}}\right)
$$

where $F_{0}$ is the pion decay constant in the chiral limit and $\phi$ is expanded in the $N^{2}-1$ traceless Hermitian basis $\phi=\lambda^{a} \phi^{a}$, with $\lambda^{a}$ and $\phi^{a}$ the $\mathrm{SU}(N)$ generators and the Goldstone boson fields, respectively. $\mathrm{U}(x)$ and $u(x)$ transform under $\mathrm{SU}(N)_{L} \times \mathrm{SU}(N)_{R}$ as

$$
U \mapsto g_{R} U g_{L}^{\dagger}, \quad u \mapsto \sqrt{g_{R} U g_{L}^{\dagger}} \equiv g_{R} u K^{\dagger}=K u g_{L}^{\dagger},
$$


where $g_{L} \in \mathrm{SU}(N)_{L}, g_{R} \in \mathrm{SU}(N)_{R}$, and $K$ is a nonlinear function of $g_{L}, g_{R}$ and $\mathrm{U}(x)$. The compensator field $K$ represents an element of the subgroup $\mathrm{SU}(N)_{V}$, and it reduces to $K=g_{L}=g_{R}$, independent of $\mathrm{U}(x)$, for a $\mathrm{SU}(N)_{V}$ transformation, i.e. when $g_{L}=g_{R}$ (see, e.g., ref. [20]). It is convenient for building up effective Lagrangians respecting the symmetry constraints to construct the matter fields so that they transform under $\mathrm{SU}(N)_{L} \times \mathrm{SU}(N)_{R}$ as

$$
P \mapsto P K^{\dagger}, \quad P^{\dagger} \mapsto K P^{\dagger}
$$

Here, we have followed the convention of ref. [19] to define $P^{\dagger}$ and $P$ in the fundamental and anti-fundamental representation of $\mathrm{SU}(N)$, respectively.

For the convenience of constructing a chirally invariant Lagrangian, we introduce chiral-covariant derivatives as

$$
D_{\mu} P^{\dagger}=\partial_{\mu} P^{\dagger}+\Gamma_{\mu} P^{\dagger}, \quad D_{\mu} P=\partial_{\mu} P+P \Gamma_{\mu}^{\dagger},
$$

with the chiral connection $\Gamma_{\mu}=\frac{1}{2}\left[u^{\dagger}\left(\partial_{\mu}-i r_{\mu}\right) u+u\left(\partial_{\mu}-i l_{\mu}\right) u^{\dagger}\right]$, and further $r_{\mu}=v_{\mu}+a_{\mu}$ and $l_{\mu}=v_{\mu}-a_{\mu}$. One can construct three other building blocks

$$
u_{\mu}=i\left[u^{\dagger}\left(\partial_{\mu}-i r_{\mu}\right) u+u\left(\partial_{\mu}-i l_{\mu}\right) u^{\dagger}\right], \quad \chi_{ \pm}=u^{\dagger} \chi u^{\dagger} \pm u \chi^{\dagger} u
$$

as well as the covariant derivatives on them such as $\nabla_{\mu} u_{\nu}=\partial_{\mu} u_{\nu}+\left[\Gamma_{\mu}, u_{\nu}\right]$. These operators transform under $\mathrm{SU}(N)_{L} \times \mathrm{SU}(N)_{R}$ as

$$
u_{\mu} \mapsto K u_{\mu} K^{\dagger}, \quad \chi_{ \pm} \mapsto K \chi_{ \pm} K^{\dagger}, \quad \nabla_{\mu} u_{\nu} \mapsto K \nabla_{\mu} u_{\nu} K^{\dagger}
$$

The power counting rules for these building blocks are

$$
\begin{aligned}
& D_{\mu} P^{(\dagger)} \sim \mathcal{O}(1), \quad D_{\mu} D_{\nu} P^{(\dagger)} \sim \mathcal{O}(1), \quad\left(D_{\mu} D^{\mu}+m^{2}\right) P^{(\dagger)} \sim \mathcal{O}(p), \\
& u_{\mu} \sim \mathcal{O}(p), \quad \chi_{ \pm} \sim \mathcal{O}\left(p^{2}\right), \quad \nabla_{\mu} u_{\nu} \sim \mathcal{O}\left(p^{2}\right) .
\end{aligned}
$$

Based on the power counting for the Feynman graphs, the calculation of relevant physical observables up to $\mathcal{O}\left(p^{3}\right)$ requires the effective chiral Lagrangian

$$
\mathcal{L}_{3}^{\mathrm{eff}}=\mathcal{L}_{\phi P}^{(1)}+\mathcal{L}_{\phi P}^{(2)}+\mathcal{L}_{\phi P}^{(3)}+\mathcal{L}_{\phi}^{(2)}+\mathcal{L}_{\phi}^{(4)} .
$$

The Lagrangians can be found in refs. [3, 17-19, 21]. For completeness, we list the relevant terms here. The Lagrangian for the matter fields is

$$
\begin{aligned}
\mathcal{L}_{\phi P}^{(1)}= & D_{\mu} P D^{\mu} P^{\dagger}-m^{2} P P^{\dagger}, \\
\mathcal{L}_{\phi P}^{(2)}= & P\left[-h_{0}\left\langle\chi_{+}\right\rangle-h_{1} \chi_{+}+h_{2}\left\langle u_{\mu} u^{\mu}\right\rangle-h_{3} u_{\mu} u^{\mu}\right] P^{\dagger} \\
& +D_{\mu} P\left[h_{4}\left\langle u_{\mu} u^{\nu}\right\rangle-h_{5}\left\{u^{\mu}, u^{\nu}\right\}\right] D_{\nu} P^{\dagger}, \\
\mathcal{L}_{\phi P}^{(3)}= & {\left[i g_{1} P\left[\chi_{-}, u_{\nu}\right] D^{\nu} P^{\dagger}+g_{2} P\left[u^{\mu}, \nabla_{\mu} u_{\nu}+\nabla_{\nu} u_{\mu}\right] D^{\nu} P^{\dagger}\right.} \\
& \left.+g_{3} P\left[u_{\mu}, \nabla_{\nu} u_{\rho}\right] D^{\mu \nu \rho} P^{\dagger}+g_{4} P \nabla_{\nu} \chi_{+} D^{\nu} P^{\dagger}+g_{5} P\left\langle\nabla_{\nu} \chi_{+}\right\rangle D^{\nu} P^{\dagger}+\text { h.c. }\right] \\
& +i \gamma_{1} D^{\mu} P f_{\mu \nu}^{+} D^{\nu} P^{\dagger}+\gamma_{2} P\left[u^{\mu}, f_{\mu \nu}^{-}\right] D^{\nu} P^{\dagger},
\end{aligned}
$$


and the Goldstone boson Lagrangians has the form

$$
\begin{aligned}
\mathcal{L}_{\phi}^{(2)}= & \frac{F_{0}^{2}}{4}\left\langle u_{\mu} u^{\mu}\right\rangle+\frac{F_{0}^{2}}{4}\left\langle\chi_{+}\right\rangle, \\
\mathcal{L}_{\phi}^{(4)}= & L_{0}\left\langle u^{\mu} u^{\nu} u_{\mu} u_{\nu}\right\rangle+L_{1}\left\langle u_{\mu} u^{\mu}\right\rangle^{2}+L_{2}\left\langle u^{\mu} u^{\nu}\right\rangle\left\langle u_{\mu} u_{\nu}\right\rangle+L_{3}\left\langle\left(u_{\mu} u^{\mu}\right)^{2}\right\rangle+L_{4}\left\langle u^{\mu} u_{\mu}\right\rangle\left\langle\chi_{+}\right\rangle \\
& +L_{5}\left\langle u^{\mu} u_{\mu} \chi_{+}\right\rangle+L_{6}\left\langle\chi_{+}\right\rangle^{2}+L_{7}\left\langle\chi_{-}\right\rangle^{2}+\frac{L_{8}}{2}\left\langle\chi_{+}^{2}+\chi_{-}^{2}\right\rangle-i L_{9}\left\langle f_{+}^{\mu \nu} u_{\mu} u_{\nu}\right\rangle \\
& +\frac{L_{10}}{4}\left\langle f_{+}^{2}-f_{-}^{2}\right\rangle+H_{1}\left\langle F_{L}^{2}+F_{R}^{2}\right\rangle+H_{2}\left\langle\chi \chi^{\dagger}\right\rangle
\end{aligned}
$$

where $D^{\mu \nu \rho}=\left\{D_{\mu},\left\{D_{\nu}, D_{\rho}\right\}\right\}, F_{L}^{\mu \nu}=\partial^{\mu} l^{\nu}-\partial^{\nu} l^{\mu}-i\left[l^{\mu}, l^{\nu}\right], F_{R}^{\mu \nu}=\partial^{\mu} r^{\nu}-\partial^{\nu} r^{\mu}-i\left[r^{\mu}, r^{\nu}\right]$, and $f_{ \pm}^{\mu \nu}=u F_{L}^{\mu \nu} u^{\dagger} \pm u^{\dagger} F_{R}^{\mu \nu} u$.

\section{One-loop generating functional}

The generating functional up to $\mathcal{O}\left(p^{3}\right)$ consists of two parts: ${ }^{3}$

$$
Z_{3}=Z_{3}^{\text {tree }}+Z_{3}^{\text {one-loop }}
$$

The tree-level part $Z_{3}^{\text {tree }}$ is given by the Lagrangians in eqs. (2.11) and (2.12). The oneloop functional $Z_{3}^{\text {one-loop }}$ can be calculated in the standard way using the background field method. To calculate the one-loop functional, we perturb the fields $\mathrm{U}(x)$ and $P(x)$ around the solutions of classical equations of motion $\bar{U}(x)$ and $\bar{P}(x)$ as

$$
U=\bar{u} e^{-i \eta} \bar{u}, \quad P=\bar{P}+h,
$$

with $\eta=\eta^{a} \lambda^{a}\left(a=1, \ldots, N^{2}-1\right)$. In the following, we will neglect the bars over the classical field configurations for brevity. Collecting the fluctuations in $\xi_{A}=\left(\frac{F_{0}}{\sqrt{2}} \eta^{a}, h_{i}\right)$, the one-loop functional can be written as a Gaussian integral over the fluctuations,

$$
\begin{aligned}
e^{i Z^{\text {one-loop }}} & =\int[d \xi] \exp \left\{-i \int d^{4} x \xi_{A}\left(\mathbb{D}_{\mu} \mathbb{D}^{\mu}+\sigma\right)^{A B} \xi_{B}^{\dagger}\right\} \\
& =\mathcal{N} \exp \left\{-\frac{1}{2} \operatorname{tr} \log \left(\mathbb{D}_{\mu} \mathbb{D}^{\mu}+\sigma\right)\right\}
\end{aligned}
$$

where $\mathcal{N}$ is a normalization constant, "tr" indicates the trace over all the spaces including the coordinate space and the $\left(N^{2}-1+N\right)$-dimension space spanned in the basis of $\xi_{A}$. The covariant derivative $\mathbb{D}_{\mu}^{A B}$ and the non-derivative term $\sigma^{A B}$ are obtained in ref. [19]. For simplicity, we only list the single-matter sectors in following:

$$
\begin{aligned}
\mathbb{D}_{\mu}^{A B} & =\delta^{A B} \partial_{\mu}+\hat{\Gamma}_{\mu}^{A B}=\left(\begin{array}{cc}
d_{\mu}^{a b} & \frac{1}{4 \sqrt{2} F_{0}}\left(P\left[u_{\mu}, \lambda^{a}\right]\right)_{j} \\
\frac{1}{4 \sqrt{2} F_{0}}\left(\left[u_{\mu}, \lambda^{b}\right] P^{\dagger}\right)_{i} & D_{\mu}^{i j}
\end{array}\right), \\
\sigma^{A B} & =\left(\begin{array}{ll}
\sigma_{11}^{a b} & \sigma_{12}^{a j} \\
\sigma_{21}^{i b} & \sigma_{22}^{i j}
\end{array}\right),
\end{aligned}
$$

\footnotetext{
${ }^{3}$ The chiral anomalous effective Wess-Zumino-Witten action $[22,23]$ is not taken into account here.
} 
where

$$
\begin{aligned}
d_{\mu}^{a b} & =\delta^{a b} \partial_{\mu}-\frac{1}{2}\left\langle\left[\lambda^{a}, \lambda^{b}\right] \Gamma_{\mu}\right\rangle-\frac{1}{8 F_{0}^{2}}\left(D_{\mu} P\left[\lambda^{a}, \lambda^{b}\right] P^{\dagger}-P\left[\lambda^{a}, \lambda^{b}\right] D_{\mu} P^{\dagger}\right), \\
\sigma_{11}^{a b} & =-\frac{1}{8}\left\langle u_{\mu}\left[\lambda^{a},\left[u^{\mu}, \lambda^{b}\right]\right]\right\rangle+\frac{1}{16}\left\langle\left\{\lambda^{a},\left\{\chi_{+}, \lambda^{b}\right\}\right\}\right\rangle+\frac{3}{32 F_{0}^{2}} P\left[u_{\mu}, \lambda^{a}\right]\left[u^{\mu}, \lambda^{b}\right] P^{\dagger}, \\
\sigma_{12}^{a j} & =-\frac{1}{4 \sqrt{2} F_{0}}\left(P\left[\nabla_{\mu} u^{\mu}, \lambda^{a}\right]\right)_{j}-\frac{3}{4 \sqrt{2} F_{0}}\left(D_{\mu} P\left[u^{\mu}, \lambda^{a}\right]\right)_{j}, \\
\sigma_{21}^{i b} & =\frac{1}{4 \sqrt{2} F_{0}}\left(\left[\nabla_{\mu} u^{\mu}, \lambda^{b}\right] P^{\dagger}\right)_{i}+\frac{3}{4 \sqrt{2} F_{0}}\left(\left[u^{\mu}, \lambda^{b}\right] D_{\mu} P^{\dagger}\right)_{i}, \\
\sigma_{22}^{i j} & =m^{2} \delta^{i j}-\frac{1}{32 F_{0}^{2}}\left(\left[u_{\mu}, \lambda^{c}\right] P^{\dagger}\right)_{i}\left(P\left[u^{\mu}, \lambda^{c}\right]\right)_{j} .
\end{aligned}
$$

To calculate the one-loop functional $Z^{\text {one-loop }}=\frac{i}{2} \operatorname{tr} \log \left(\mathbb{D}_{\mu} \mathbb{D}^{\mu}+\sigma\right)$, we split the differential operator $\mathcal{D}=\left(\mathbb{D}_{\mu} \mathbb{D}^{\mu}+\sigma\right)$ into the Klein-Gordon operator $\mathcal{D}_{0}$ for free fields and the remaining interaction part $\delta_{r}: \mathcal{D}^{A B}=\mathcal{D}_{0}^{A B}+\delta_{r}^{A B}$. The one-loop functional can be calculated in an expansion of the interaction term:

$$
\begin{aligned}
Z^{\text {one-loop }} & =\frac{i}{2} \operatorname{tr} \log \left(\mathcal{D}_{0}+\delta_{r}\right)=\frac{i}{2} \operatorname{tr}\left[\log \mathcal{D}_{0}+\log \left(\mathbf{1}-\delta_{r} \Delta\right)\right] \\
& =-\frac{i}{2} \operatorname{tr}\left(\delta_{r} \Delta\right)-\frac{i}{4} \operatorname{tr}\left(\delta_{r} \Delta \delta_{r} \Delta\right)-\frac{i}{6} \operatorname{tr}\left(\delta_{r} \Delta \delta_{r} \Delta \delta_{r} \Delta\right)+\ldots
\end{aligned}
$$

where we omit the irrelevant constant term $\frac{i}{2} \operatorname{tr} \log \left(\mathcal{D}_{0}\right), \Delta$ is the inverse of $-\mathcal{D}_{0}$ :

$$
\Delta^{A B}(x-y)=\delta^{A B} \int \frac{d^{d} p}{(2 \pi)^{d}} \frac{e^{-i p(x-y)}}{p^{2}-m_{A}^{2}+i \epsilon},
$$

and the remainder $\delta_{r}$ is

$$
\delta_{r}=\left\{\hat{\Gamma}^{\mu}, \partial_{\mu}\right\}+\hat{\Gamma}^{\mu} \hat{\Gamma}_{\mu}+\hat{\sigma},
$$

with $\hat{\sigma}^{A B}=\sigma^{A B}-m_{A}^{2} \delta^{A B}$. The first term $\operatorname{tr}\left(\delta_{r} \Delta\right)$ in eq. (3.7) is the set of all tadpole graphs. The second term collects all the two-point-loop graphs, etc. Since the external fields $v_{\mu}$ and $s\left(a_{\mu}\right.$ and $p$ ) correspond to terms with an even (odd) number of boson fields, following the counting scheme used in refs. [2,3], we count $v_{\mu}$ and $s-\mathcal{M}$ as $\mathcal{O}\left(\phi^{2}\right)$, where $\phi$ here should be understood as representing both the Goldstone boson and matter fields. Thus, $\hat{\Gamma}_{\mu}$ and $\hat{\sigma}$ are of $\mathcal{O}\left(\phi^{2}\right)$. The the one-loop functional up to $\mathcal{O}\left(\phi^{4}\right)$, i.e. with at most four external meson fields, can be calculated as [24]

$$
\begin{aligned}
Z^{\text {one-loop }}= & -\frac{i}{2} \operatorname{tr}\left[\sigma(x) \Delta(0)+\hat{\Gamma}^{\mu}(x) \hat{\Gamma}_{\mu}(x) \Delta(0)\right] \\
& -\frac{i}{4} \operatorname{tr}\left[\left\{\hat{\Gamma}^{\mu}(x), \partial_{\mu}^{x}\right\} \Delta(x-y)\left\{\hat{\Gamma}^{\nu}(y), \partial_{\nu}^{y}\right\} \Delta(y-x)\right. \\
& +2\left\{\hat{\Gamma}^{\mu}(x), \partial_{\mu}^{x}\right\} \Delta(x-y) \hat{\sigma}(y) \Delta(y-x) \\
& \left.+2 \hat{\Gamma}^{\mu}(x) \hat{\Gamma}_{\mu}(x) \Delta(x-y) \hat{\sigma}(y) \Delta(y-x)\right]+\mathcal{O}\left(\phi^{6}\right)
\end{aligned}
$$




$$
\begin{aligned}
= & -\frac{i}{2} \operatorname{tr}\left[\hat{\sigma}(x) \Delta(0)+\hat{\Gamma}^{\mu}(x) \hat{\Gamma}_{\mu}(x) \Delta(0)\right] \\
& -\frac{i}{4} \operatorname{tr}\left[\hat{\Gamma}^{\mu}(x) \partial_{\mu}^{x} \Delta(x-y) \hat{\Gamma}^{\nu}(y) \partial_{\nu}^{y} \Delta(y-x)\right. \\
& +\hat{\Gamma}^{\mu}(x) \partial_{\nu}^{x} \Delta(x-y) \hat{\Gamma}^{\nu}(y) \partial_{\mu}^{y} \Delta(y-x) \\
& +\hat{\Gamma}^{\mu}(x) \partial_{\mu}^{x} \partial_{\nu}^{x} \Delta(x-y) \hat{\Gamma}^{\nu}(y) \Delta(y-x) \\
& +\hat{\Gamma}^{\mu}(x) \Delta(x-y) \hat{\Gamma}^{\nu}(y) \partial_{\mu}^{y} \partial_{\nu}^{y} \Delta(y-x) \\
& +2 \hat{\Gamma}^{\mu}(x) \partial_{\mu}^{x} \Delta(x-y) \hat{\sigma}(y) \Delta(y-x) \\
& +2 \hat{\Gamma}^{\mu}(x) \Delta(x-y) \hat{\sigma}(y) \partial_{\mu}^{y} \Delta(y-x) \\
& +\hat{\sigma}(x) \Delta(x-y) \hat{\sigma}(y) \Delta(y-x)]+\mathcal{O}\left(\phi^{6}\right) .
\end{aligned}
$$

When the light quark masses are different, the free Klein-Gordon propagator is not diagonal in the cartesian basis spanned by $\lambda^{1}, \lambda^{2}, \ldots, \lambda^{N^{2}-1}$. It is more convenient to use the physical basis such as $\lambda^{\pi^{+}}=-\frac{1}{\sqrt{2}}\left(\lambda^{1}+i \lambda^{2}\right)$ and so on. More explicitly, the one-loop functional to $\mathcal{O}\left(\phi^{4}\right)$ can be written as [3]

$$
\begin{aligned}
Z^{\text {one-loop }}= & \frac{i}{2} \sum_{P} \int d^{4} x \Delta_{P}(0) \hat{\sigma}_{P P}(x)+\frac{i}{4} \sum_{P, Q}\left[\Delta_{P}(0)+\Delta_{Q}(0)\right] \hat{\Gamma}_{\mu P Q}(x) \hat{\Gamma}_{Q P}^{\mu}(x) \\
& +\sum_{P, Q} \int d^{4} x d^{4} y\left[M_{\mu \nu}^{P Q}(x-y) \hat{\Gamma}_{P Q}^{\mu}(x) \hat{\Gamma}_{Q P}^{\nu}(y)+K_{\mu}^{P Q}(x-y) \hat{\Gamma}_{P Q}^{\mu}(x) \hat{\sigma}_{Q P}(y)\right. \\
& \left.+J^{P Q}(x-y) \hat{\sigma}_{P Q}(x) \hat{\sigma}_{Q P}(y)\right]+\mathcal{O}\left(\phi^{6}\right)
\end{aligned}
$$

where

$$
\begin{aligned}
M_{\mu \mu}^{P Q}(z) & =\frac{i}{4}\left(\partial_{\mu} \Delta_{P} \partial_{\nu} \Delta_{Q}+\partial_{\nu} \Delta_{P} \partial_{\mu} \Delta_{Q}-\partial_{\mu \nu} \Delta_{P} \Delta_{Q}-\Delta_{P} \partial_{\mu \nu} \Delta_{Q}\right), \\
K_{\mu}^{P Q}(z) & =\frac{i}{2}\left(\partial_{\mu} \Delta_{P} \Delta_{Q}-\Delta_{P} \partial_{\mu} \Delta_{Q}\right), \\
J^{P Q}(z) & =-\frac{i}{4} \Delta_{P} \Delta_{Q},
\end{aligned}
$$

with $\Delta_{P}(z)=\Delta\left(z, M_{P}^{2}\right)$ defined as the free propagator for a spinless field of mass $M_{P}$ in $d$ dimensions. The explicit expressions of the various kernels in terms of loop integrals are listed in appendix. A.

\section{Subtraction of the power counting breaking terms}

In calculations using dimensional regularization with the $\overline{\mathrm{MS}}$ scheme, any loop integral involving matter field propagators contains terms starting from $\mathcal{O}(1)$, in contrast with the power counting in eq. (2.1). Thus, there are PCB terms which contribute at orders lower than that required by eq. (2.1), and they will be calculated and subtracted in this section.

The loops involving only matter field propagators do not play any dynamical role in the low-energy effective field theory, neither do they introduce non-analyticity in the quark masses, and thus can be absorbed into a redefinition of LECs. As a result, the 
explicit closed matter field loops are not necessary to be included in the calculation. Thus, the Goldstone boson part of the one-loop functional is identical to that in the standard Goldstone boson ChPT, which can be found in refs. [3]. The PCB terms of interest are from the loops containing both Goldstone boson and matter field internal propagators. They correspond to the terms of $M_{\mu \nu}^{P Q}, K_{\mu}^{P Q}$ and $J^{P Q}$ in eq. (3.10) with $P$ and $Q$ in different blocks (Goldstone bosons and matter fields). Since we are only interested in the single matter field sector, which is the sector relevant for processes with a single matter field in both initial and final states and can be studied using the effective Lagragians in eq. (2.11), and the terms contributing at orders lower than that required by eq. (2.1), we only need the following terms of $\hat{\Gamma}_{\mu}$ and $\hat{\sigma}$ for calculating the PCB part, which is of $\mathcal{O}\left(p^{2}\right)$, of the one-loop generating functional

$$
\begin{aligned}
\hat{\Gamma}_{\mu}^{A B} & =\frac{1}{4 \sqrt{2} F_{0}}\left(\begin{array}{cc}
0 & \left(P\left[u_{\mu}, \lambda^{a}\right]\right)_{j} \\
\left(\left[u_{\mu}, \lambda^{b}\right] P^{\dagger}\right)_{i} & 0
\end{array}\right), \\
\hat{\sigma}^{\prime A B} & =\frac{3}{4 \sqrt{2} F_{0}}\left(\begin{array}{cc}
0 & -\left(D^{\mu} P\left[u_{\mu}, \lambda^{a}\right]\right)_{j} \\
\left(\left[u_{\mu}, \lambda^{b}\right] D^{\mu} P^{\dagger}\right)_{i} & 0
\end{array}\right) .
\end{aligned}
$$

Since the elements of $\Gamma_{\mu}^{\prime}$ and $\sigma^{\prime}$ are of $\mathcal{O}(p)$, the PCB terms of loops only refer to the $\mathcal{O}(1)$ terms of the loop integrals. As a result, they are independent of the internal Goldstone boson masses. Therefore, the relevant one-loop generating functional to $\mathcal{O}\left(\phi^{4}\right)$ can be rewritten in the form

$$
\begin{aligned}
Z^{\text {one-loop }} & \int d^{4} x d^{4} y \frac{d^{4} p}{(2 \pi)^{4}} e^{-i p(x-y)} \\
& \times\left\{\frac{1}{8}\left\langle\hat{\Gamma}^{\prime \mu \nu}(x) \hat{\Gamma}_{\mu \nu}^{\prime}(y)\right\rangle\left[B_{0}\left(p^{2}, m^{2}, 0\right)+4 B_{1}\left(p^{2}, m^{2}, 0\right)+4 B_{11}\left(p^{2}, m^{2}, 0\right)\right]\right. \\
& -\left\langle\hat{\Gamma}_{\mu}^{\prime}(x) \hat{\Gamma}^{\prime \mu}(y)\right\rangle\left[B_{00}\left(p^{2}, m^{2}, 0\right)+\frac{p^{2}}{4}\left(B_{0}\left(p^{2}, m^{2}, 0\right)+4 B_{1}\left(p^{2}, m^{2}, 0\right)+4 B_{11}\left(p^{2}, m^{2}, 0\right)\right)\right] \\
& \left.+\left\langle\partial_{\mu} \tilde{\Gamma}^{\prime \mu}(x) \hat{\sigma}(y)\right\rangle\left[\frac{B_{0}\left(p^{2}, m^{2}, 0\right)}{2}+B_{1}\left(p^{2}, m^{2}, 0\right)\right]+\frac{1}{4} B_{0}\left(p^{2}, m^{2}, 0\right)\left\langle\hat{\sigma}^{\prime}(x) \hat{\sigma}^{\prime}(y)\right\rangle\right\}, \\
= & \int d^{4} x d^{4} y \frac{d^{4} p}{(2 \pi)^{4}} e^{-i p(x-y)}\left\{-\left\langle\hat{\Gamma}_{\mu}^{\prime}(x) \hat{\Gamma}^{\prime \mu}(y)\right\rangle B_{00}\left(p^{2}, m^{2}, 0\right)\right. \\
& -\frac{1}{4}\left\langle\partial_{\mu} \Gamma_{\nu}^{\prime} \partial^{\nu} \Gamma^{\prime \mu}\right\rangle\left[B_{0}\left(p^{2}, m^{2}, 0\right)+4 B_{1}\left(p^{2}, m^{2}, 0\right)+4 B_{11}\left(p^{2}, m^{2}, 0\right)\right] \\
& \left.+\left\langle\partial_{\mu} \tilde{\Gamma}^{\prime \mu}(x) \hat{\sigma}(y)\right\rangle\left[\frac{B_{0}\left(p^{2}, m^{2}, 0\right)}{2}+B_{1}\left(p^{2}, m^{2}, 0\right)\right]+\frac{1}{4} B_{0}\left(p^{2}, m^{2}, 0\right)\left\langle\hat{\sigma}^{\prime}(x) \hat{\sigma}^{\prime}(y)\right\rangle\right\},
\end{aligned}
$$

where we have defined $\hat{\Gamma}_{\mu \nu}^{\prime}=\partial_{\mu} \hat{\Gamma}_{\nu}^{\prime}-\partial_{\nu} \hat{\Gamma}_{\mu}^{\prime}+\left[\hat{\Gamma}_{\mu}^{\prime}, \hat{\Gamma}_{\nu}^{\prime}\right]$, and

$$
\tilde{\Gamma}_{\mu}^{\prime A B}=\frac{1}{4 \sqrt{2} F_{0}}\left(\begin{array}{cc}
0 & \left(P\left[u_{\mu}, \lambda^{a}\right]\right)_{j} \\
-\left(\left[u_{\mu}, \lambda^{b}\right] P^{\dagger}\right)_{i} & 0
\end{array}\right) .
$$

The expressions for the loop functions $B_{0}\left(s, m^{2}, M^{2}\right), B_{1}\left(s, m^{2}, M^{2}\right), B_{00}\left(s, m^{2}, M^{2}\right)$ and $B_{11}\left(s, m^{2}, M^{2}\right)$ are given in appendix A. 
Since all the operators in eq. (4.2) are of $\mathcal{O}\left(p^{2}\right)$, we can extract the PCB terms by keeping only the $\mathcal{O}\left(p^{0}\right)$ part of these loop functions. In the EOMS scheme, the PCB terms come from the leading chiral expansion of one-loop functions $A\left(m^{2}\right)$ and $B_{0}\left(m^{2}, m^{2}, 0\right)$, as shown in appendix B. Applying the results of appendix B and the equations of motion for the classical background fields, it is easy to obtain the PCB terms of interest:

$$
\begin{aligned}
Z_{\text {one-loop }}^{\text {PCB }}= & \frac{1}{16 \pi^{2} F_{0}^{2}} \int d^{4} x\left\{\frac{m^{2}}{144}\left[2-3 \log \left(\frac{m^{2}}{\mu^{2}}\right)\right]\left\langle P P^{\dagger}\right\rangle\left\langle u_{\mu} u^{\mu}\right\rangle\right. \\
& +\frac{m^{2} N}{144}\left[2-3 \log \left(\frac{m^{2}}{\mu^{2}}\right)\right]\left\langle P u_{\mu} u^{\mu} P^{\dagger}\right\rangle \\
& +\frac{7}{72}\left[5-3 \log \left(\frac{m^{2}}{\mu^{2}}\right)\right]\left\langle D^{\mu} P D^{\nu} P^{\dagger}\right\rangle\left\langle u_{\mu} u_{\nu}\right\rangle \\
& \left.+\frac{7 N}{144}\left[5-3 \log \left(\frac{m^{2}}{\mu^{2}}\right)\right]\left\langle D^{\mu} P\left\{u_{\mu}, u_{\nu}\right\} D^{\nu} P^{\dagger}\right\rangle\right\} .
\end{aligned}
$$

One can subtract these PCB terms in eq. (4.4) to get a consistent power counting. Within the EOMS scheme, they are absorbed into the redefinition of the LECs of $\mathcal{O}\left(p^{2}\right)$ as

$$
\mathcal{L}_{\phi P}^{(2)}=\sum_{i=0}^{5} h_{i} \mathcal{O}_{i}=\sum_{i=0}^{5}\left[h_{i}^{r}(\mu)+h_{i}^{0} \lambda+\frac{1}{16 \pi^{2} F_{0}^{2}} h_{i}^{\mathrm{PCB}}\right] \mathcal{O}_{i},
$$

where $\mathcal{O}_{i}$ represent local operators in the Lagrangian of $\mathcal{O}\left(p^{2}\right), \mu$ is the scale of dimensional regularization, $\lambda=\mu^{d-4}(4 \pi)^{-d / 2} /(d-4), h_{i}^{r}(\mu)$ are the UV finite and scale-dependent part of the LECs $h_{i}$, the coefficients $h_{i}^{0}$ of the UV divergence $\sim \lambda$ have been calculated in ref. [19], and $h_{i}^{\mathrm{PCB}}$ are the PCB parts. From eq. (2.11) and eq. (4.4), they can be easily read off as

$$
\begin{array}{lll}
h_{0}^{\mathrm{PCB}}=0, & h_{2}^{\mathrm{PCB}}=-m^{2}\left(\frac{1}{72}-\frac{1}{48} \log \frac{m^{2}}{\mu^{2}}\right), & h_{3}^{\mathrm{PCB}}=m^{2}\left(\frac{N}{72}-\frac{N}{48} \log \frac{m^{2}}{\mu^{2}}\right), \\
h_{1}^{\mathrm{PCB}}=0, & h_{4}^{\mathrm{PCB}}=-\frac{7}{72}\left(5-3 \log \frac{m^{2}}{\mu^{2}}\right), & h_{5}^{\mathrm{PCB}}=\frac{7 N}{144}\left(5-3 \log \frac{m^{2}}{\mu^{2}}\right) .
\end{array}
$$

Note that we have dropped the tadpole loops with matter field propagators completely. For $N=3$, the expressions of $h_{i}^{\mathrm{PCB}}$ agree with those found in an explicit calculation of the charmed-meson-Goldstone-boson scattering amplitudes [18].

\section{Summary}

In this paper, we have given the explicit generating functional for Green functions of at most four external fields in a chiral effective field theory for a single matter field up to $\mathcal{O}\left(p^{3}\right)$. In the case we considered, the matter fields are spinless and in the fundamental representation of $\mathrm{SU}(N)$. We have derived the power counting breaking terms in the oneloop generating functional up to $\mathcal{O}\left(p^{3}\right)$ using dimensional regularization. In the EOMS scheme, they are subtracted from the loop integrals and absorbed into a redefinition of $\mathcal{O}\left(p^{2}\right)$ LECs. The framework can be used for any theories with spontaneous symmetry 
breaking of $\mathrm{SU}(N)_{L} \times \mathrm{SU}(N)_{R}$ to $\mathrm{SU}(N)_{V}$ with spinless matter fields in fundamental representation. Examples in QCD of the matter fields are ground state pseudoscalar mesons except for the pions such as kaons and heavy mesons.

\section{Acknowledgments}

UGM acknowledges the warm hospitality of the ITP of CAS where part of this work was done. This work is supported in part by DFG and NSFC through funds provided to the Sino-German CRC 110 "Symmetries and the Emergence of Structure in QCD" (NSFC Grant No. 11621131001), by the Thousand Talents Plan for Young Professionals, by the Chinese Academy of Sciences (CAS) (Grant No. QYZDB-SSW-SYS013), and by the CAS President's International Fellowship Initiative (PIFI) (Grant No. 2015VMA076).

\section{A Kernels}

The kernels $\mathcal{K}_{a}^{P Q}(x-y)$ including $M_{\mu \nu}^{P Q}(x-y), K_{\mu}^{P Q}(x-y)$ and $J^{P Q}(x-y)$ in eq. (3.10) have the form

$$
\mathcal{K}_{a}^{P Q}(x-y)=\int \frac{d^{4} p}{(2 \pi)^{2}} e^{-i p(x-y)} \mathcal{K}_{a}^{P Q}(p)
$$

with

$$
\begin{aligned}
M_{\mu \nu}^{P Q}(p)= & -g_{\mu \nu} B_{00}\left(p^{2}, m_{P}^{2}, m_{Q}^{2}\right) \\
& -\frac{1}{4} p_{\mu} p_{\nu}\left[B_{0}\left(p^{2}, m_{P}^{2}, m_{Q}^{2}\right)+4 B_{1}\left(p^{2}, m_{P}^{2}, m_{Q}^{2}\right)+4 B_{11}\left(p^{2}, m_{P}^{2}, m_{Q}^{2}\right)\right] \\
K_{\mu}^{P Q}(p)= & i p_{\mu}\left[\frac{1}{2} B_{0}\left(p^{2}, m_{P}^{2}, m_{Q}^{2}\right)+B_{1}\left(p^{2}, m_{P}^{2}, m_{Q}^{2}\right)\right] \\
J^{P Q}(p)= & \frac{1}{4} B_{0}\left(p^{2}, m_{P}^{2}, m_{Q}^{2}\right),
\end{aligned}
$$

where the loop functions $A, B_{0}, B_{1}, B_{00}$ and $B_{11}$ are defined through:

$$
\begin{aligned}
A\left(m^{2}\right) & =\frac{\mu^{4-d}}{i} \int \frac{d^{d} k}{(2 \pi)^{d}} \frac{1}{k^{2}-m^{2}+i \epsilon}, \\
B_{0}\left(p^{2}, m^{2}, M^{2}\right) & =\frac{\mu^{4-d}}{i} \int \frac{d^{d} k}{(2 \pi)^{d}} \frac{1}{\left(k^{2}-m^{2}+i \epsilon\right)\left[(k+p)^{2}-M^{2}+i \epsilon\right]} \\
p^{\mu} B_{1}\left(p^{2}, m^{2}, M^{2}\right) & =\frac{\mu^{4-d}}{i} \int \frac{d^{d} k}{(2 \pi)^{d}} \frac{k^{\mu}}{\left(k^{2}-m^{2}+i \epsilon\right)\left[(k+p)^{2}-M^{2}+i \epsilon\right]}, \\
g^{\mu \nu} B_{00}\left(p^{2}, m^{2}, M^{2}\right) & +p^{\mu} p^{\nu} B_{11}\left(p^{2}, m^{2}, M^{2}\right) \\
& =\frac{\mu^{4-d}}{i} \int \frac{d^{d} k}{(2 \pi)^{d}} \frac{k^{\mu} k^{\nu}}{\left(k^{2}-m^{2}+i \epsilon\right)\left[(k+p)^{2}-M^{2}+i \epsilon\right]}
\end{aligned}
$$


The expressions for these loop functions in dimensional regularization are

$$
\begin{aligned}
A_{0}\left(m^{2}\right)= & \frac{m^{2}}{16 \pi^{2}}\left(R+\log \frac{m^{2}}{\mu^{2}}\right), \\
B_{0}\left(p^{2}, m^{2}, M^{2}\right)= & \frac{1}{16 \pi^{2}}\left[-R+1-\log \frac{M^{2}}{\mu^{2}}\right. \\
& \left.+\frac{\Delta+p^{2}}{2 p^{2}} \log \frac{M^{2}}{m^{2}}+\frac{p^{2}-(m-M)^{2}}{p^{2}} \rho\left(p^{2}\right) \log \frac{\rho\left(p^{2}\right)-1}{\rho\left(p^{2}\right)+1}\right], \\
B_{1}\left(p^{2}, m^{2}, M^{2}\right)= & \frac{1}{2 p^{2}}\left[A_{0}\left(m^{2}\right)-A_{0}\left(M^{2}\right)-\left(p^{2}+\Delta\right) B_{0}\left(p^{2}, m^{2}, M^{2}\right)\right], \\
B_{00}\left(p^{2}, m^{2}, M^{2}\right)= & -\frac{1}{288 \pi^{2}}\left(p^{2}-3 \Sigma\right)+\frac{1}{12 p^{2}}\left\{\left(p^{2}+\Delta\right) A_{0}\left(m^{2}\right)+\left(p^{2}-\Delta\right) A_{0}\left(M^{2}\right)\right. \\
& \left.+\left[4 p^{2} m^{2}-\left(p^{2}+\Delta\right)^{2}\right] B_{0}\left(p^{2}, m^{2}, M^{2}\right)\right\} \\
B_{11}\left(p^{2}, m^{2}, M^{2}\right)= & \frac{1}{288 \pi^{2} p^{2}}\left(p^{2}-3 \Sigma\right)+\frac{1}{3 p^{4}}\left\{-\left(p^{2}+\Delta\right) A_{0}\left(m^{2}\right)+\left(2 p^{2}+\Delta\right) A_{0}\left(M^{2}\right)\right. \\
& \left.-\left[p^{2} m^{2}-\left(p^{2}+\Delta\right)^{2}\right] B_{0}\left(p^{2}, m^{2}, M^{2}\right)\right\},
\end{aligned}
$$

where we have defined $R=\frac{2}{d-4}+\gamma_{E}-1-\log 4 \pi$, with $\gamma_{E}$ the Euler constant, $\Delta=m^{2}-M^{2}$, $\Sigma=m^{2}+M^{2}$, and

$$
\rho=\sqrt{\frac{p^{2}-(m+M)^{2}}{p^{2}-(m-M)^{2}}} .
$$

\section{B Infrared regular parts of loop integrals}

Using the method proposed in refs. [10, 25], we derive the infrared regular parts of loop integrals to $\mathcal{O}\left(p^{2}\right)$. Only the leading order, i.e. $\mathcal{O}\left(p^{0}\right)$, part is used to extract the PCB terms. The closed matter loop $A_{0}\left(m^{2}\right)$ is infrared regular, and the regular part of the loop integral $B_{0}\left(p^{2}, m^{2}, M^{2}\right)$ can be expanded as [25]

$$
\begin{aligned}
B_{0}^{\text {reg. }}\left(p^{2}, m^{2}, M^{2}\right)= & \frac{\Gamma(2-d / 2)}{(4 \pi)^{d / 2}(d-3)}\left(\frac{m}{\mu}\right)^{d-4}\left[1-\frac{p^{2}-m^{2}}{2 m^{2}}+\frac{(d-6)\left(p^{2}-m^{2}\right)^{2}}{4 m^{4}(d-5)}\right. \\
& \left.+\frac{(d-3) M^{2}}{2 m^{2}(d-5)}+\cdots\right] .
\end{aligned}
$$

More explicitly, using eq. (A.8), the infrared regular PCB parts of the loop functions are

$$
\begin{aligned}
A_{0}^{\mathrm{PCB}}\left(m^{2}\right) & =-\frac{m^{2}}{16 \pi^{2}} \log \frac{m^{2}}{\mu^{2}}, \\
B_{0}^{\mathrm{PCB}}\left(p^{2}, m^{2}, 0\right) & =\frac{1}{16 \pi^{2}}\left(1-\log \frac{m^{2}}{\mu^{2}}\right), \\
B_{1}^{\mathrm{PCB}}\left(p^{2}, m^{2}, 0\right) & =-\frac{1}{16 \pi^{2}}\left(1-\frac{1}{2} \log \frac{m^{2}}{\mu^{2}}\right), \\
B_{00}^{\mathrm{PCB}}\left(p^{2}, m^{2}, 0\right) & =\frac{m^{2}}{288 \pi^{2}}\left(2-3 \log \frac{m^{2}}{\mu^{2}}\right), \\
B_{11}^{\mathrm{PCB}}\left(p^{2}, m^{2}, 0\right) & =\frac{1}{144 \pi^{2}}\left(8-3 \log \frac{m^{2}}{\mu^{2}}\right) .
\end{aligned}
$$


Open Access. This article is distributed under the terms of the Creative Commons Attribution License (CC-BY 4.0), which permits any use, distribution and reproduction in any medium, provided the original author(s) and source are credited.

\section{References}

[1] S. Weinberg, Phenomenological Lagrangians, Physica A 96 (1979) 327 [InSPIRE].

[2] J. Gasser and H. Leutwyler, Chiral Perturbation Theory to One Loop, Annals Phys. 158 (1984) 142 [INSPIRE].

[3] J. Gasser and H. Leutwyler, Chiral Perturbation Theory: Expansions in the Mass of the Strange Quark, Nucl. Phys. B 250 (1985) 465 [INSPIRE].

[4] S.L. Adler, Axial vector vertex in spinor electrodynamics, Phys. Rev. 177 (1969) 2426 [INSPIRE].

[5] G.A. Christos, Chiral Symmetry and the U(1) Problem, Phys. Rept. 116 (1984) 251 [INSPIRE].

[6] J. Gasser, M.E. Sainio and A. Svarc, Nucleons with Chiral Loops, Nucl. Phys. B 307 (1988) 779 [INSPIRE].

[7] A. Roessl, Pion kaon scattering near the threshold in chiral SU(2) perturbation theory, Nucl. Phys. B 555 (1999) 507 [hep-ph/9904230] [INSPIRE].

[8] E.E. Jenkins and A.V. Manohar, Baryon chiral perturbation theory using a heavy fermion Lagrangian, Phys. Lett. B 255 (1991) 558 [INSPIRE].

[9] V. Bernard, N. Kaiser, J. Kambor and U.-G. Meißner, Chiral structure of the nucleon, Nucl. Phys. B 388 (1992) 315 [inSPIRE].

[10] T. Becher and H. Leutwyler, Baryon chiral perturbation theory in manifestly Lorentz invariant form, Eur. Phys. J. C 9 (1999) 643 [hep-ph/9901384] [INSPIRE].

[11] T. Fuchs, J. Gegelia, G. Japaridze and S. Scherer, Renormalization of relativistic baryon chiral perturbation theory and power counting, Phys. Rev. D 68 (2003) 056005 [hep-ph/0302117] [INSPIRE].

[12] V. Bernard, N. Kaiser and U.-G. Meißner, Chiral dynamics in nucleons and nuclei, Int. J. Mod. Phys. E 4 (1995) 193 [hep-ph/9501384] [INSPIRE].

[13] V. Bernard, N. Kaiser, U.-G. Meißner and A. Schmidt, Aspects of nucleon Compton scattering, Z. Phys. A 348 (1994) 317 [hep-ph/9311354] [InSPIRE].

[14] H.-B. Tang, A new approach to chiral perturbation theory for matter fields, hep-ph/9607436 [INSPIRE].

[15] P.J. Ellis and H.-B. Tang, Pion nucleon scattering in a new approach to chiral perturbation theory, Phys. Rev. C 57 (1998) 3356 [hep-ph/9709354] [INSPIRE].

[16] J. Gegelia, G.S. Japaridze and K.S. Turashvili, Calculation of loop integrals by dimensional counting, Theor. Math. Phys. 101 (1994) 1313 [INSPIRE].

[17] F.-K. Guo, C. Hanhart and U.-G. Meißner, Interactions between heavy mesons and Goldstone bosons from chiral dynamics, Eur. Phys. J. A 40 (2009) 171 [arXiv:0901.1597] [InSPIRE]. 
[18] D.-L. Yao, M.-L. Du, F.-K. Guo and U.-G. Meißner, One-loop analysis of the interactions between charmed mesons and Goldstone bosons, JHEP 11 (2015) 058 [arXiv:1502.05981] [INSPIRE].

[19] M.-L. Du, F.-K. Guo and U.-G. Meißner, One-loop renormalization of the chiral Lagrangian for spinless matter fields in the $\mathrm{SU}(N)$ fundamental representation, arXiv:1607.00822 [INSPIRE].

[20] G. Ecker, Chiral perturbation theory, Prog. Part. Nucl. Phys. 35 (1995) 1 [hep-ph/9501357] [INSPIRE].

[21] J. Bijnens, Chiral perturbation theory beyond one loop, Prog. Part. Nucl. Phys. 58 (2007) 521 [hep-ph/0604043] [INSPIRE].

[22] J. Wess and B. Zumino, Consequences of anomalous Ward identities, Phys. Lett. B 37 (1971) 95 [INSPIRE].

[23] E. Witten, Current Algebra, Baryons and Quark Confinement, Nucl. Phys. B 223 (1983) 433 [INSPIRE].

[24] R. Unterdorfer and G. Ecker, Generating functional for strong and nonleptonic weak interactions, JHEP 10 (2005) 017 [hep-ph/0507173] [INSPIRE].

[25] M.R. Schindler, J. Gegelia and S. Scherer, Infrared and extended on mass shell renormalization of two loop diagrams, Nucl. Phys. B 682 (2004) 367 [hep-ph/0310207] [INSPIRE]. 